\title{
Early use of nitazoxanide in mild COVID-19 disease: randomised, placebo-controlled trial
}

\author{
Patricia R.M. Rocco $\mathbb{1}^{1,14}$, Pedro L. Silva $\mathbb{1}^{1,14}$, Fernanda F. Cruz ${ }^{1,14}$, Marco Antonio C. Melo-Junior ${ }^{2}$, \\ Paulo F.G.M.M. Tierno ${ }^{3}$, Marcos A. Moura ${ }^{4}$, Luís Frederico G. De Oliveira ${ }^{5}$, Cristiano C. Lima ${ }^{6}$, \\ Ezequiel A. Dos Santos $\mathbb{1}^{7}$, Walter F. Junior ${ }^{8}$, Ana Paula S.M. Fernandes ${ }^{9}$, Kleber G. Franchini ${ }^{10}$, \\ Erick Magri ${ }^{2}$, Nara F. de Moraes ${ }^{3}$, José Mário J. Gonçalves ${ }^{4}$, Melanie N. Carbonieri ${ }^{5}$, Ivonise S. Dos Santos ${ }^{6}$, \\ Natália F. Paes ${ }^{7}$, Paula V.M. Maciel ${ }^{8}$, Raissa P. Rocha ${ }^{9}$, Alex F. de Carvalho ${ }^{9}$, Pedro Augusto Alves ${ }^{11}$, \\ José Luiz Proença-Módena $\mathbb{1}^{12}$, Artur T. Cordeiro ${ }^{10}$, Daniela B.B. Trivella ${ }^{10}$, Rafael E. Marques $\mathbb{(}^{10}$, \\ Ronir R. Luiz ${ }^{1}$, Paolo Pelosi ${ }^{13}$ and Jose Roberto Lapa e Silva ${ }^{1}$ on behalf of the SARITA-2 investigators
}

\begin{abstract}
${ }^{1}$ Federal University of Rio de Janeiro, Rio de Janeiro, Brazil. ${ }^{2}$ Hospital Municipal de Emergências Albert Sabin, São Caetano, Brazil. ${ }^{3}$ Hospital Municipal de Barueri Dr Francisco Moran, Barueri, Brazil. ${ }^{4}$ Hospital e Maternidade Therezinha de Jesus, Juiz de Fora, Brazil. ${ }^{5}$ Hospital Santa Casa de Misericórdia de Sorocaba, Sorocaba, Brazil. ${ }^{6}$ Secretaria de Estado de Saúde do Distrito Federal, Brasília, Brazil. ${ }^{7}$ Secretaria Municipal de Saúde de Bauru, Bauru, Brazil. ${ }^{8}$ Secretaria Municipal de Saúde de Guarulhos, Guarulhos, Brazil. ${ }^{9}$ Centro de Tecnologia de Vacinas, Universidade Federal de Minas Gerais, Belo Horizonte, Brazil. ${ }^{10}$ Brazilian Biosciences National Laboratory (LNBio) and Brazilian Center for Research in Energy and Materials (CNPEM), Campinas, Brazil. ${ }^{11}$ Instituto René Rachou Fiocruz, Belo Horizonte, Brazil. ${ }^{12}$ University of Campinas, UNICAMP, Campinas, Brazil. ${ }^{13}$ Dept of Surgical Sciences and Integrated Diagnostics, University of Genoa, Genoa, Italy. ${ }^{14}$ These authors contributed equally.
\end{abstract}

Corresponding author: Patricia R.M. Rocco (prmrocco@biof.ufrj.br)

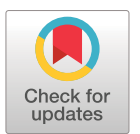

Copyright OERS 2021

This version is distributed under the terms of the Creative Commons Attribution Non-Commercial Licence 4.0.

This article has supplementary material available from erj. ersjournals.com

Received: 5 Oct 2020 Accepted: 4 Dec 2020

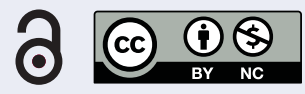

Shareable abstract (@ERSpublications)

This was the first study to evaluate the effect of early nitazoxanide therapy in mild COVID-19. Nitazoxanide did not accelerate symptom resolution after 5 days of therapy, but did reduce viral load significantly with no serious adverse events. https://bit.ly/37i75pr

Cite this article as: Rocco PRM, Silva PL, Cruz FF, et al. Early use of nitazoxanide in mild COVID-19 disease: randomised, placebo-controlled trial. Eur Respir J 2021; 58: 2003725 [DOI: 10.1183/ 13993003.03725-2020].

\section{Abstract}

Background Nitazoxanide is widely available and exerts broad-spectrum antiviral activity in vitro. However, there is no evidence of its impact on severe acute respiratory syndrome coronavirus 2 (SARSCoV-2) infection.

Methods In a multicentre, randomised, double-blind, placebo-controlled trial, adult patients presenting up to 3 days after onset of coronavirus disease 2019 (COVID-19) symptoms (dry cough, fever and/or fatigue) were enrolled. After confirmation of SARS-CoV-2 infection using reverse transcriptase PCR on a nasopharyngeal swab, patients were randomised 1:1 to receive either nitazoxanide (500 mg) or placebo, three times daily, for 5 days. The primary outcome was complete resolution of symptoms. Secondary outcomes were viral load, laboratory tests, serum biomarkers of inflammation and hospitalisation rate. Adverse events were also assessed.

Results From June 8 to August 20, 2020, 1575 patients were screened. Of these, 392 (198 placebo, 194 nitazoxanide) were analysed. Median (interquartile range) time from symptom onset to first dose of study drug was 5 (4-5) days. At the 5-day study visit, symptom resolution did not differ between the nitazoxanide and placebo arms. Swabs collected were negative for SARS-CoV-2 in $29.9 \%$ of patients in the nitazoxanide arm versus $18.2 \%$ in the placebo arm $(p=0.009)$. Viral load was reduced after nitazoxanide compared to placebo $(\mathrm{p}=0.006)$. The percentage viral load reduction from onset to end of therapy was higher with nitazoxanide (55\%) than placebo $(45 \%)(p=0.013)$. Other secondary outcomes were not significantly different. No serious adverse events were observed.

Conclusions In patients with mild COVID-19, symptom resolution did not differ between nitazoxanide and placebo groups after 5 days of therapy. However, early nitazoxanide therapy was safe and reduced viral load significantly. 
Introduction

The majority of patients infected with the severe acute respiratory syndrome coronavirus 2 (SARS-CoV-2) present mild symptoms of coronavirus disease 2019 (COVID-19) and recover with supportive care; however, in certain hosts, even mild disease may progress to clinical deterioration or a protracted course [1]. To date, no therapeutic interventions have proven effective in mild COVID-19.

Drug repurposing has been recognised as an important tool in the search for effective COVID-19 therapies, as it reduces development costs and timelines [2]. Nitazoxanide, a clinically approved and commercially available antiparasitic drug, has been found to have broad-spectrum antiviral activity, including against coronaviruses, influenza viruses and hepatitis B and C viruses [3]. Furthermore, it has been shown to inhibit SARS-CoV-2 replication at low micromolar concentrations in Vero CCL81 cells [4]. In addition, nitazoxanide is orally bioavailable and broadly well-tolerated, thus representing a promising alternative for the management of COVID-19 were it to prove effective in vivo [3]. Efficacy in early treatment (initiated soon after symptom onset to reduce viral load and prevent disease progression) would be particularly appealing from a public health standpoint. However, there is no evidence of its safety or efficacy as therapy for mild COVID-19 patients.

In this context, a multicentre, randomised, placebo-controlled trial was performed to evaluate whether early nitazoxanide therapy would be effective in accelerating symptom resolution. Secondarily, viral load, markers of inflammation, hospitalisation rate and the safety of nitazoxanide as compared with placebo were assessed.

\section{Methods}

\section{Study design}

A double-blind, placebo-controlled trial was conducted at five freestanding urgent care centres and two hospitals across Brazil (supplementary table S1). The trial was designed by the executive committee and approved by the Brazilian National Commission for Research Ethics (CAAE: 32258920.0.1001.5257) and individual ethics committees of the participating sites. The trial was funded by the Brazilian Ministry of Science, Technology, and Innovation via the National Council for Scientific and Technological Development. Nitazoxanide was provided free of charge by Eurofarma, which had no further role in the design or conduct of the trial. The executive committee assures the accuracy of the data and fidelity of the trial to the protocol, which was registered in the Brazilian Registry of Clinical Trials (REBEC; number RBR-4nr86m) and ClinicalTrials.gov (identifier number NCT04552483). The independent data and safety monitoring board, composed of experts in clinical trials and infectious diseases, was convened after 25\%, $50 \%$ and $75 \%$ of the participants had completed 14 days of follow-up and had access to information on adverse events and efficacy outcomes at every quartile.

The trial was conducted in accordance with the principles of the Declaration of Helsinki and the International Council for Harmonisation Good Clinical Practice guideline (E6R2). Online clinical monitoring and quality control were outsourced to a contract research organisation (ATCGen, Campinas, Brazil). This report follows the Consolidated Standards of Reporting Trials guideline [5]. The final protocol, amendments and changes to the trial protocol, and the final statistical analysis plan are detailed in the supplementary methods.

\section{Patients}

Consecutive adult patients (aged $\geqslant 18$ years) who presented with clinical symptoms of COVID-19 (defined for the purposes of this trial as dry cough, fever and/or fatigue) of no longer than 3 days' duration were enrolled. The exclusion criteria were negative reverse-transcriptase quantitative real-time PCR (RT-PCR) test for SARS-CoV-2 on a nasopharyngeal swab specimen; inability to swallow; pre-existing conditions precluding the safe conduct of study procedures, including severe renal, heart, respiratory, liver or autoimmune diseases, cancer in the past 5 years, or known allergy or hypersensitivity to nitazoxanide; therapy with nitazoxanide in the 30 days before presentation; and clinical suspicion of bacterial pneumonia or tuberculosis.

\section{Randomisation and masking}

Patients were randomly assigned (1:1) using a computer-generated random number list to receive either placebo or nitazoxanide (500 $\mathrm{mg}$ oral solution, $20 \mathrm{mg} \cdot \mathrm{mL}^{-1}(25 \mathrm{~mL})$, three times daily for 5 days), dispensed by the pharmacy of each study site. Placebo and nitazoxanide were colour-matched to ensure that assessors were unaware of group allocation at all time points. 


\section{Procedures}

On day 0 (baseline), patients were assessed for eligibility. Informed consent was obtained from each patient or, if the patient was unable to provide consent, from a healthcare proxy. A nasopharyngeal swab was then collected for RT-PCR testing. In order to mitigate any bias, all RT-PCR analyses were processed centrally at CT-Vacinas (Federal University of Minas Gerais, Belo Horizonte, Brazil); specimens were sent on the day of collection by commercial courier. Patients who tested positive for SARS-CoV-2 (result obtained 1-2 days after RT-PCR) (figure 1) were contacted by telephone and asked to return to the health facility to which they had originally presented. Site investigators then performed a comprehensive physical examination and measured body temperature, heart and respiratory rates, blood pressure, and peripheral oxygen saturation. Ethnicity, current medications and date of symptom onset were self-reported by patients. Blood was then drawn for measurement of complete blood count, C-reactive protein (CRP) and serum biomarkers of inflammation (interleukin (IL)-6, IL-8, IL-1 $\beta$, tumour necrosis factor- $\alpha$ and interferon- $\gamma$ ) (see supplementary methods for details of RT-PCR testing and biomarker assessment).

All patients took home a symptom journal designed to gather information on daily symptoms, new symptoms and the date of resolution of each symptom (supplementary table S2). Study data were entered directly into an electronic database by an assigned staff member at each study site and further validated by external trial monitoring staff at ATCGen.

1 day after completion of therapy, patients returned to the study sites to return their symptom journals and provide a new nasopharyngeal sample for RT-PCR and blood samples for complete blood count, CRP and serum biomarkers. Data validation was undertaken exclusively by the designated study site manager, with assistance from ATCGen staff as needed.

\section{Primary outcome}

The primary outcome was complete resolution of the three symptoms of interest (dry cough, fever and/or fatigue) after 5 days of therapy.

\section{Secondary outcomes}

Secondary outcomes were reduction in viral RNA load on nasopharyngeal swab specimens (from baseline until the day after completion of therapy); improvement in laboratory parameters (including serum biomarkers of inflammation); and incidence of hospital admission after completion of therapy. Patients who did not return to the study sites either to receive the therapy or after the end of therapy were contacted by telephone to understand the reasons for nonadherence and were then excluded from per-protocol analysis (supplementary methods). Adverse events, regardless of grade, were monitored throughout the trial by review of the electronic medical record, physical examination, vital signs and laboratory tests from

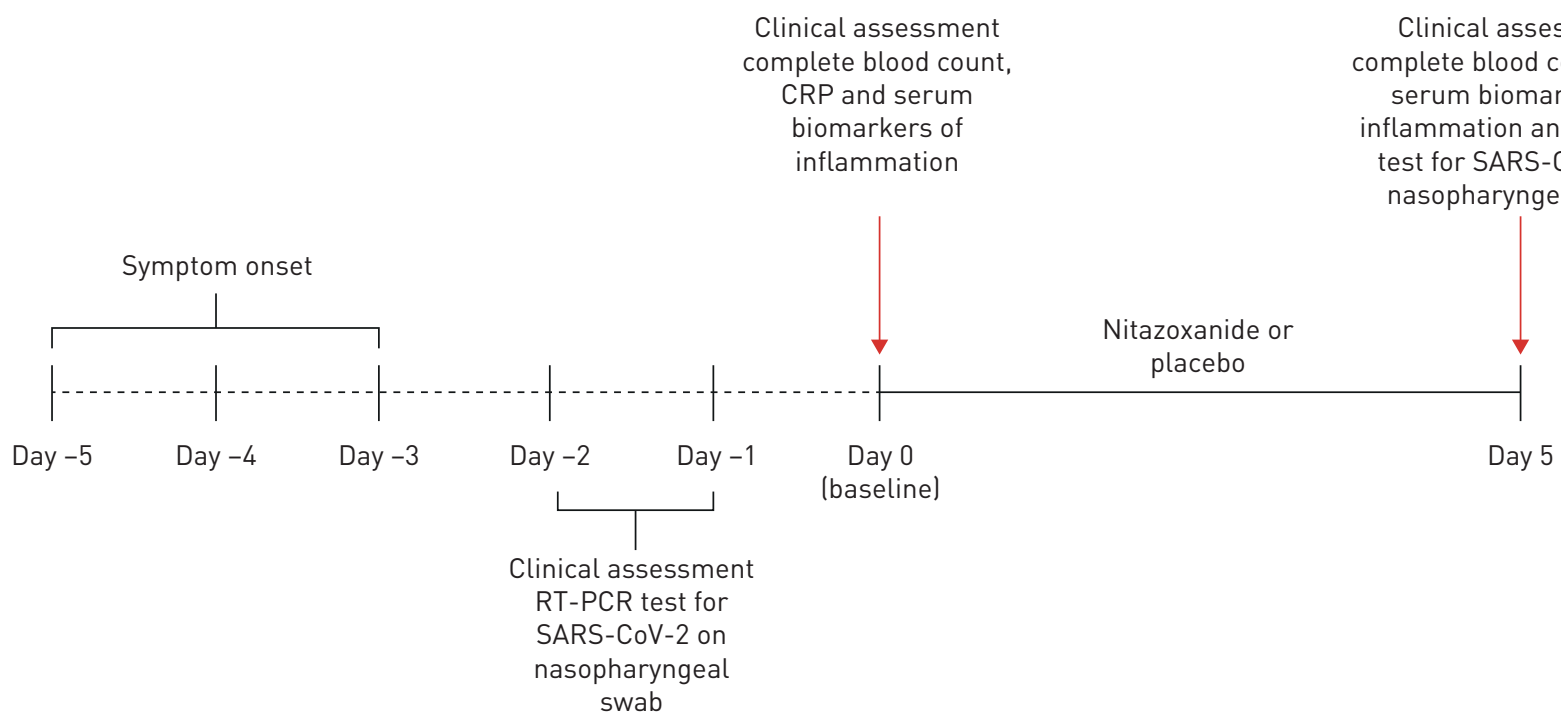

FIGURE 1 Timeline of study design. RT-PCR: real-time reverse-transcriptase PCR; SARS-CoV-2: severe acute respiratory syndrome coronavirus 2; CRP: C-reactive protein. 
enrolment through day 14. The Medical Dictionary for Regulatory Activities (MedDRA, version 23.0) was used for classification.

\section{Statistical analyses}

We estimated that a sample size of 392 patients (196 per arm; allocation ratio 1:1) would provide 85\% power to detect an $11 \%$ increase in symptom-free days after nitazoxanide initiation compared to placebo at a two-sided significance level of $\alpha=0.05$. Descriptive statistics were used for demographic, laboratory and clinical data. For qualitative variables, Fisher's exact test was performed. The Mann-Whitney U-test and the Wilcoxon test were used for between- and within-groups comparisons, respectively. Statistical analyses were performed in the SPSS version 27 environment (IBM, Armonk, NY, USA), and a two-tailed p-value $<0.05$ was considered significant (supplementary methods).

\section{Results}

Between June 8 and August 20, 2020, 1575 patients were assessed for eligibility at the study sites. Of these, 475 tested positive for SARS-CoV-2 infection by RT-PCR and underwent randomisation. Figure 2 summarises the enrolment and follow-up of study participants. Reasons for exclusion before randomisation included negative RT-PCR collected at day 0 and/or absence of COVID-19 symptoms ( $\mathrm{n}=1062)$, refusal to participate $(n=27)$, hospitalisation before the first dose of therapy $(n=5)$ and other reasons $(n=6)$. After randomisation $(n=475)$, patients were excluded due to discontinued intervention $(n=41)$, moderate adverse events $(n=7)$ and hospitalisation $(n=10)$. Six patients in the nitazoxanide arm and one patient in the placebo arm discontinued therapy due to moderate diarrhoea and vomiting within the first 2 days; both had already experienced improvement of COVID-19 symptoms. 10 patients (five from each arm) were hospitalised due to clinical deterioration; none had completed the 5-day course of therapy. Two patients, both in the nitazoxanide arm, required intensive care unit admission (supplementary table S3). During analysis, 12 patients were excluded from the nitazoxanide arm and 15 from the placebo arm due to protocol deviation, missing data on the primary outcome, or non-evaluability, resulting in a studied population of 392 patients (194 in the nitazoxanide arm and 198 in the placebo arm).

\section{Baseline characteristics}

Patients' characteristics at baseline are given in table 1. Demographics and clinical characteristics were balanced in both groups. Age ranged from 18 to 77 years. Just over half (53\%) were female and 69\% were white. Regarding timing of therapy initiation, $8 \%$ of patients were enrolled on day $1,25 \%$ on day 2 , and $67 \%$ on day 3 after symptom onset. The median (interquartile range (IQR)) time between symptom onset and first dose of nitazoxanide or placebo was 5 (4-5) days. Fever, dry cough and fatigue were the most frequent clinical features present at enrolment. At baseline, the overall median (IQR) viral load in the nasopharyngeal swab at baseline did not differ significantly between the two arms. Additional characteristics of the study population at baseline are given in supplementary table S4.

\section{Primary outcome}

Complete resolution of symptoms (dry cough, fever and fatigue) did not differ between the nitazoxanide and placebo arms after 5 days of therapy (figure 3, supplementary figure S2).

\section{Secondary outcome}

After 5 days of therapy, viral load was lower in the nitazoxanide arm compared to placebo ( $\mathrm{p}=0.006$ ) (table 2). Moreover, the percentage of reduction in viral load from day 0 (baseline) (table 1) to day 5 (table 2) of therapy was significantly higher in the nitazoxanide arm (55\%) than in the placebo arm (45\%) ( $p=0.013$ ). At the end-of-therapy visit, $29.9 \%$ of patients in the nitazoxanide arm versus $18.2 \%$ in the placebo arm were negative for SARS-CoV-2 on RT-PCR ( $p=0.009)$ (table 2). Vital signs (supplementary table S4), total leukocyte, neutrophil, lymphocyte and platelet counts, CRP levels, and serum biomarkers of inflammation (table 2) did not differ between baseline and day 5 of therapy in the nitazoxanide arm, nor between the nitazoxanide and placebo arms. No patients who completed 5-day course of nitazoxanide were hospitalised (supplementary table S3).

No deaths or life-threatening adverse events were reported in either arm. Mild and moderate adverse events were experienced by patients in both arms (nitazoxanide 30.9\%, placebo 30.4\%) during the 5-day course of therapy (table 3). The most common adverse events (grades 1 and 2) were diarrhoea, headache and nausea, with no significant differences between groups. Four patients reported severe adverse events (headache alone or with diarrhoea); however, they were more frequent in the placebo arm. Discoloured urine is a known side-effect of nitazoxanide therapy, which might raise concerns about inadvertent unblinding. However, although this event was indeed more frequent in the treatment arm, it was also reported by patients receiving placebo (5.6\% versus 1.5\%) (table 3). 


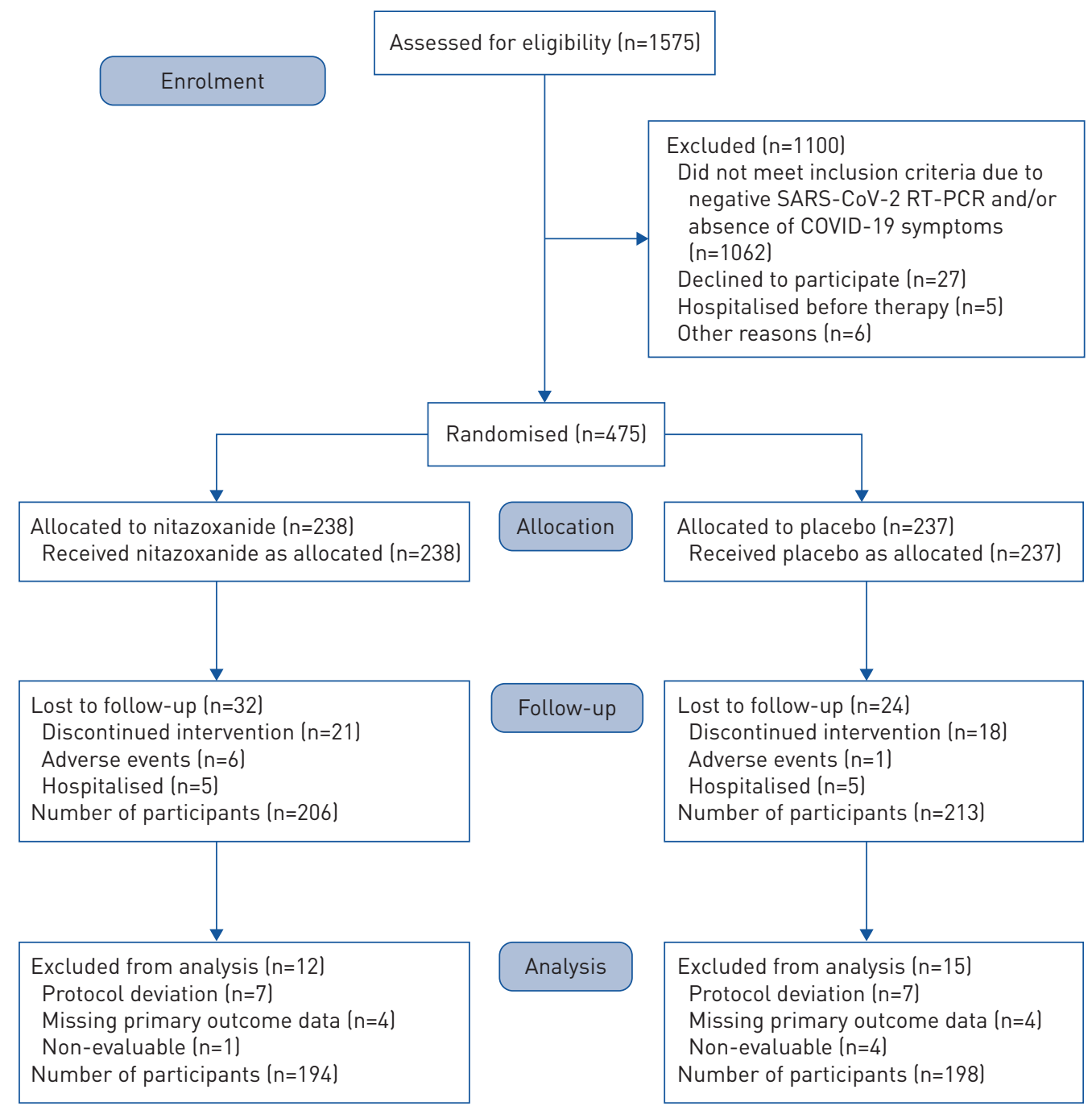

FIGURE 2 Enrolment, randomisation, follow-up and treatment. 1575 patients were assessed for eligibility at the study sites. Of these, 475 tested positive for severe acute respiratory syndrome coronavirus 2 (SARS-CoV-2) infection by reverse transcriptase (RT)-PCR and underwent randomisation. Reasons for exclusion before randomisation included negative RT-PCR collected at day 0 (baseline) and/or absence of coronavirus disease 2019 (COVID-19) symptoms ( $n=1062)$, refusal to participate $(n=27)$, hospitalisation before the first dose of therapy $(n=5)$ and other reasons $(n=6)$. After randomisation $(n=475)$, patients were excluded due to discontinued intervention ( $n=39)$, moderate adverse events $(n=7)$ (all gastrointestinal upset) and hospitalisation ( $n=10)(n=5$ patients from each group, none of whom completed therapy). During analysis, 12 patients were excluded from the nitazoxanide arm and 15 from the placebo arm due to protocol deviation, missing data on the primary outcome or non-evaluability, resulting in a studied population of 392 patients (194 in the nitazoxanide arm and 198 in the placebo arm).

\section{Discussion}

In this multicentre, double-blind, randomised, placebo-controlled trial of patients with mild COVID-19, we found that symptom resolution (dry cough, fever and fatigue) did not differ between the nitazoxanide and placebo after 5 days of therapy. Nitazoxanide was safe, significantly reduced viral load and increased the proportion of patients testing negative for SARS-CoV-2 after 5 days of therapy compared to placebo. Nitazoxanide did not prevent hospitalisation or effect any change in complete blood count, CRP levels or serum biomarkers of inflammation. Moreover, nitazoxanide is inexpensive, widely available, and, to date, no other recommended therapies have been demonstrated to provide any benefit in this population.

There is an urgent need for evidence-based pharmacotherapeutics for COVID-19. In the challenging context of a pandemic, drug repurposing can reduce the time-consuming drug development process and 
TABLE 1 Characteristics of the participants testing positive for severe acute respiratory syndrome coronavirus 2 on reverse transcriptase (RT)-PCR at baseline

\begin{tabular}{|c|c|c|c|}
\hline & Nitazoxanide & Placebo & $\mathrm{p}$-value \\
\hline Participants & 194 & 198 & \\
\hline Age range years & & & 0.891 \\
\hline $18-39$ & $115(59)$ & $113(57)$ & \\
\hline $40-59$ & $68(35)$ & $74(37)$ & \\
\hline $60-77$ & $11(6)$ & $11(6)$ & \\
\hline Sex & & & 0.054 \\
\hline Male & $101(52)$ & $83(42)$ & \\
\hline Female & $93(48)$ & $115(58)$ & \\
\hline Ethnicity & & & 0.644 \\
\hline White & $131(68)$ & $138(70)$ & \\
\hline Asian & $5(3)$ & $2(1)$ & \\
\hline Indigenous Brazilian & $0(0)$ & $1(0.5)$ & \\
\hline Black & $31(16)$ & $32(16)$ & \\
\hline Mixed & 27 (14) & $24(12)$ & \\
\hline $\mathrm{BMI} \mathrm{kg} \cdot \mathrm{m}^{-2}$ & & & 1.000 \\
\hline$<29.9$ & $134(69)$ & $136(69)$ & \\
\hline$\geqslant 30.0$ & $60(31)$ & $62(31)$ & \\
\hline Comorbidities & & & 0.091 \\
\hline No & $171(88)$ & $162(82)$ & \\
\hline Yes ${ }^{\#}$ & $23(12)$ & $36(18)$ & \\
\hline Time from onset of symptoms to enrolment days & & & 0.241 \\
\hline 1 & $14(7)$ & $18(9)$ & \\
\hline 2 & $42(22)$ & $55(28)$ & \\
\hline 3 & $138(71)$ & $125(63)$ & \\
\hline Time from onset of symptoms to the first dose of nitazoxanide or placebo, days & $5(4-5)$ & $5(4-5)$ & 0.124 \\
\hline \multicolumn{4}{|l|}{ Concomitant medications } \\
\hline None & $154(80)$ & $158(80)$ & 1.000 \\
\hline Angiotensin-II receptor antagonists & $6(3)$ & $12(6)$ & 0.227 \\
\hline Angiotensin-conversing enzyme inhibitors & $1(1)$ & $1(0.5)$ & 0.833 \\
\hline Metformin & $1(0.5)$ & $4(2)$ & 0.372 \\
\hline Statins & $0(0)$ & $4(2)$ & 0.123 \\
\hline \multicolumn{4}{|l|}{ Symptoms at baseline } \\
\hline \multicolumn{4}{|l|}{ Inclusion criteria } \\
\hline Dry cough & $141(73)$ & $148(75)$ & 0.574 \\
\hline Fever & $109(56)$ & $115(58)$ & 0.760 \\
\hline Fatigue & $95(49)$ & $95(48)$ & 0.920 \\
\hline \multicolumn{4}{|l|}{ Secondary symptoms } \\
\hline Sore throat & $82(42)$ & $68(34)$ & 0.119 \\
\hline Myalgia & $60(31)$ & $53(27)$ & 0.374 \\
\hline Headache & $64(33)$ & $81(41)$ & 0.117 \\
\hline Anosmia & $17(9)$ & $18(9)$ & 1.000 \\
\hline Ageusia & $20(10)$ & $17(9)$ & 0.607 \\
\hline Diarrhoea & $14(7)$ & $5(3)$ & 0.035 \\
\hline $\begin{array}{l}\text { Nasopharyngeal swab RT-PCR viral load } \log _{10} \\
\text { copies } \cdot \mathrm{mL}^{-1}\end{array}$ & $7.06(5.77-8.13)$ & $7.49(6.15-8.32)$ & 0.065 \\
\hline $\mathrm{S}_{\mathrm{pO}_{2}} \%$ & $97.3 \pm 1.4$ & $97.4 \pm 1.3$ & 0.835 \\
\hline $\mathrm{WBC} \times 10^{3}$ cells $\cdot \mathrm{mL}^{-1}$ & $5.4(4.3-6.6)$ & $5.3(4.6-6.5)$ & 0.904 \\
\hline Neutrophils $\times 10^{3}$ cells $\cdot \mathrm{mL}^{-1}$ & $2.8(2.1-3.9)$ & $2.9(2.2-4.0)$ & 0.984 \\
\hline Lymphocytes $\times 10^{3}$ cells $\cdot \mathrm{mL}^{-1}$ & $1.9(1.5-2.2)$ & $1.8(1.4-2.4)$ & 0.423 \\
\hline Platelets $\times 10^{3}$ cells $\cdot \mathrm{mL}^{-1}$ & $245(205-245)$ & $213(177-257)$ & 0.243 \\
\hline $\mathrm{CRP} \mathrm{mg} \cdot \mathrm{L}^{-1}$ & $6.0(2.0-15.0)$ & $4.5(2.0-12.2)$ & 0.190 \\
\hline \multicolumn{4}{|l|}{ Cytokine concentration $\mathrm{pg} \cdot \mathrm{dL}^{-1}$} \\
\hline IL-6 & $0(0-4.10)$ & $0(0-8.34)$ & 0.471 \\
\hline IL-8 & $2.79(0-12.48)$ & $5.51(0.38-17.91)$ & 0.090 \\
\hline IL-1 $\beta$ & $0(0-0)$ & $0(0-19.45)$ & 0.088 \\
\hline TNF- $\alpha$ & $0(0-0)$ & $0(0-0)$ & 0.179 \\
\hline IFN- $\gamma$ & $0(0-16.24)$ & $0(0-10.24)$ & 0.526 \\
\hline
\end{tabular}

Data are presented as $\mathrm{n}, \mathrm{n}(\%)$, median (interquartile range) or mean \pm sD, unless otherwise stated. BMI: body mass index; $S_{\mathrm{pO}_{2}}$ : peripheral oxyhaemoglobin saturation; WBC: white blood cells; CRP: C-reactive protein; IL: interleukin; TNF: tumour necrosis factor; IFN: interferon. ": systemic arterial hypertension, diabetes mellitus, asthma. No significant differences were observed between the two groups. 


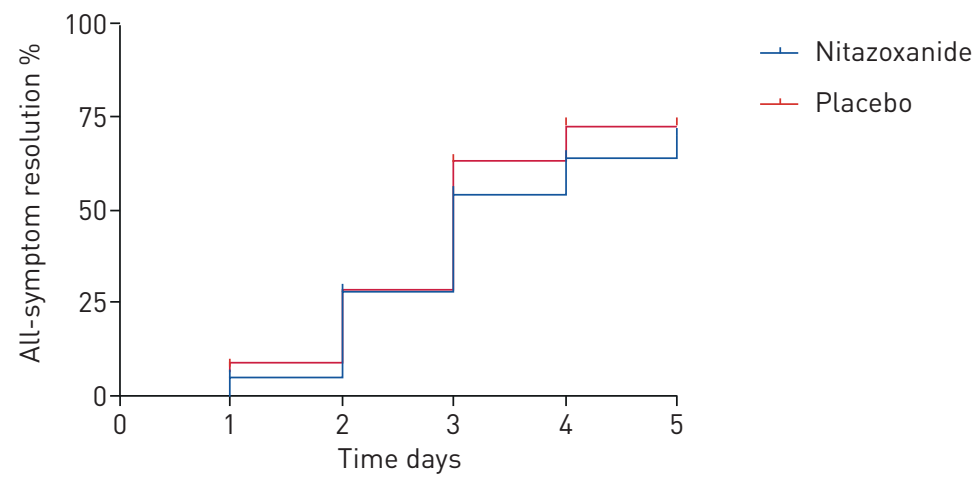

Patients free of symptoms $n$

$\begin{array}{lllllll}\text { Nitazoxanide } & 0 & 10 & 54 & 106 & 125 & 135\end{array}$

$\begin{array}{lllllll}\text { Placebo } & 0 & 16 & 56 & 125 & 144 & 146\end{array}$

Log-rank test $p=0.277$

FIGURE 3 Kaplan-Meier curve of symptom resolution after 5 days of therapy.

allow rapid deployment of effective therapies to the population [2]. Before implementation of this trial, the National Institutes of Health Clinical Collection library, a collection of 727 United States Food and Drug Administration (FDA)-approved drugs or drug-like compounds with a history of use in human clinical trials, was screened for potential in vitro antiviral activity against SARS-CoV-2 (supplementary figure S1). Nitazoxanide and tizoxanide, its active metabolite, significantly reduced viral load in Vero E6, human embryonic kidney (HEK 293T) and lung epithelial (Calu-3) cells infected with SARS-CoV-2, without inducing loss of cell viability. The adverse effect profile of nitazoxanide is well known, since it has been commercially available and used in the clinic since 1996; indeed, a commercial formulation was used in this trial. A dosage regimen of $500 \mathrm{mg}$ every $12 \mathrm{~h}$ is approved and commonly prescribed for treatment of intestinal parasitosis [5], with few reported adverse events. In our study, the same dose was administered, but every $8 \mathrm{~h}$, based on in vitro pharmacological studies by our group and published data on plasma concentration [6-8], in order to maximise potential inhibition of SARS-CoV-2 in vivo [7]. We assumed that high ratios of maximum serum concentration at doses safe in humans to maximal in vitro effective antiviral concentration would translate into higher potential to achieve viral suppression at approved doses [9].

TABLE 2 Secondary outcomes after 5 days of therapy

\begin{tabular}{|c|c|c|c|}
\hline & Nitazoxanide & Placebo & $\mathrm{p}$-value \\
\hline Participants & 194 & 198 & \\
\hline Nasopharyngeal swab RT-PCR viral load $\log _{10}$ copies $\cdot \mathrm{mL}^{-1}$ & $3.63(0-5.03)$ & $4.13(2.88-5.31)$ & 0.006 \\
\hline RT-PCR status & & & 0.009 \\
\hline Positive & $136(70.0)$ & $162(82.8)$ & \\
\hline Negative & 58 (29.9) & 36 (18.2) & \\
\hline $\mathrm{WBC} \times 10^{3}$ cells $\cdot \mathrm{mL}^{-1}$ & $6.1(5.1-7.3)$ & $6.4(5.3-7.8)$ & 0.080 \\
\hline Neutrophils $\times 10^{3}$ cells $\cdot \mathrm{mL}^{-1}$ & $3.4(2.6-4.4)$ & $3.6(2.7-4.7)$ & 0.327 \\
\hline Lymphocytes $\times 10^{3}$ cells $\cdot \mathrm{mL}^{-1}$ & $2.1(1.7-2.5)$ & $2.2(1.7-2.6)$ & 0.078 \\
\hline Platelets $\times 10^{3}$ cells $\cdot \mathrm{mL}^{-1}$ & $240(209-288)$ & $239(198-285)$ & 0.275 \\
\hline CRP $\mathrm{mg} \cdot \mathrm{L}^{-1}$ & $5.0(1.0-16.2)$ & $4.5(2.0-13.0)$ & 0.445 \\
\hline \multicolumn{4}{|l|}{ Cytokine concentration $\mathrm{pg} \cdot \mathrm{dL}^{-1}$} \\
\hline IL-6 & $0(0-0.03)$ & $0(0-0.03)$ & 0.992 \\
\hline IL-8 & $2.73(0-12.24)$ & $2.70(0-11.42)$ & 0.855 \\
\hline IL-1 $1 \beta$ & $0(0-0)$ & $0(0-0)$ & 0.399 \\
\hline TNF- $\alpha$ & $0(0-0)$ & $0(0-0)$ & 0.627 \\
\hline IFN- $\gamma$ & $0(0-12.54)$ & $0(0-3.25)$ & 0.286 \\
\hline
\end{tabular}


TABLE 3 Adverse events

\begin{tabular}{lccc}
\hline & Nitazoxanide & Placebo & p-value \\
\hline Participants & 194 & 198 & \\
Participants with at least one adverse event & $60(30.9)$ & $60(30.4)$ & 0.913 \\
\hline Participants with two adverse events & $22(11.3)$ & $18(9.1)$ & 0.507 \\
\hline Participants with three or more adverse events & $16(8.2)$ & $12(6.1)$ & 0.438 \\
\hline Participants with severe adverse event & $1(0.5)$ & $1(0.5)$ & 0.999 \\
\hline Participants discontinuing treatment because of moderate & $6(3.1)$ & $1(0.5)$ & 0.065 \\
$\quad$ adverse events & & & \\
Detailed adverse events & & & \\
$\quad$ Diarrhoea & $57(29.4)$ & $49(24.7)$ & 0.309 \\
\hline Headache & $34(17.5)$ & $32(16.1)$ & 0.787 \\
\hline Nausea & $28(14.4)$ & $29(14.6)$ & 0.999 \\
\hline Abdominal pain & $10(5.2)$ & $5(2.5)$ & 0.197 \\
\hline Abnormal colour of urine & $11(5.6)$ & $3(1.5)$ & 0.031 \\
\hline Vomiting & $9(4.6)$ & $3(1.5)$ & 0.085 \\
\hline Pruritus & $4(2.1)$ & $1(0.5)$ & 0.212 \\
\hline Urticaria & $1(0.5)$ & $3(1.5)$ & 0.623 \\
\hline
\end{tabular}

Data are presented as $\mathrm{n}$ or $\mathrm{n}(\%)$, unless otherwise stated, and include all adverse events reported after nitazoxanide or placebo.

Nitazoxanide failed to meet the primary outcome in patients with mild COVID-19 when evaluated after 5 days of therapy. Consistent with the in vitro data, we observed significant reductions in viral load after a 5-day course of nitazoxanide in patients with mild COVID-19. This effect may have epidemiological impact, potentially decreasing community spread of SARS-CoV-2 [9, 10], morbidity [11] and mortality [12].

We enrolled patients with mild COVID-19 as soon as possible after symptom onset because antivirals seem to be more effective at this stage of infection [13]. The cut-off point for enrolment was 3 days after onset of first symptom; however, as therapy was begun only after confirmation of SARS-CoV-2 infection, the median timing of treatment initiation was 5 (4-5) days after symptom onset (table 1). In previous trials of putative antiviral agents, median time to initiation of treatment was 13 days [14], 9 days [15], 8-9 days [16] and 4-5 days [17] after symptom onset. Initiating therapy even earlier might be more effective, since the peak SARS-CoV-2 viral load usually occurs before symptom onset [18] and systemic hyperinflammation rather than viral pathogenicity dominates later stages of COVID-19, at which point antiviral therapy could be ineffective $[18,19]$. Additionally, analyses were performed 5 days after therapy, since a longer period of time would result in symptom resolution in most patients independent of treatment; in previous studies, the median to time from onset to resolution of symptoms has been reported as 8 (6.25-11.5) days [20].

Besides timing, two additional points which may have biased our findings in favour of the nitazoxanide arm were the severity of COVID-19 at presentation and the favourable demographic profile of the sample. Only patients with mild COVID-19 were enrolled; most were young adults (aged 18-39 days), few had comorbidities (12-18\%) and use of concomitant medications was infrequent ( $<20 \%$ of the sample). Further studies are needed to evaluate whether nitazoxanide may play a role in more advanced COVID-19. In this line, Hung et al. [17] recently reported the importance of combining antiviral therapies in patients with severe COVID-19, aiming to reduce viral load and mitigate symptoms.

The increase in dosage frequency of nitazoxanide did not result in any significant change in the adverse event rate. Neither frequency nor severity of adverse events differed significantly between the arms, except for urine discolouration (a known and harmless effect), suggesting that nitazoxanide is safe for patients with mild COVID-19.

This study has several limitations. Only three symptoms (dry cough, fever and fatigue) were considered for analysis of primary outcome, even though COVID-19 is now known to have protean manifestations, and patients reported other symptoms. Patients were followed during the 5-day course of therapy; however, a long-term analysis of the effects of therapy should be performed. All patients were instructed to take their study medication three times daily as prescribed, return to the study site if symptoms worsened or adverse effects developed and complete their symptom journals with data on any adverse events, the intensity of each COVID-19 symptom, and when symptoms abated and resolved. However, we cannot ascertain the 
extent to which patients followed these instructions. Nevertheless, given the placebo-controlled design, nonadherence may have occurred in both groups. In the nitazoxanide group, $\sim 3 \%$ stopped therapy due to gastrointestinal upset after COVID-19 symptoms had already improved. Finally, no chest computed tomography scans or plain radiographs were obtained in these patients, since our work was mostly performed at freestanding mobile urgent care centres.

In summary, in patients with mild COVID-19 enrolled within 3 days of symptom onset, nitazoxanide as compared with placebo was not an effective therapy in terms of accelerating symptom resolution after 5 days of therapy, and did not modify clinically relevant secondary outcomes. However, nitazoxanide was safe, significantly decreased viral load and increased the proportion of patients who tested negative for SARS-CoV-2 after completion of therapy.

Acknowledgements: The authors thank Moira Elizabeth Schöttler (Rio de Janeiro, Brazil) and Filippe Vasconcellos (São Paulo, Brazil) for editing assistance.

ClinicalTrials.gov number NCT04552483. Qualified clinical researchers can request access to de-identified participant dataset, informed consent forms and related documents, including the study protocol. that underlie this article through submission of a proposal with a valuable research question to the corresponding author, subject to agreement of a contract.

Author contributions: P.R.M. Rocco, P.L. Silva and F.F. Cruz were responsible for the design, analysis and writing of the manuscript. M.A.C.M. Junior, P.F.G.M.M. Tierno, M.A. Moura, L.F.G. De Oliveira, C.C. Lima, E.A. Dos Santos, W.F. Junior, E. Magri, N.F. de Moraes, J.M.J Gonçalves, M.N. Carbonieri, I.S. Dos Santos, N.F. Paes and P.V.M. Maciel were responsible for recruitment and clinical care of the patients. A.P.S.M. Fernandes, K.G. Franchini, R.P. Rocha, A.F. de Carvalho, P.A. Alves, J.L.P. Modena, A.T. Cordeiro, D.B.B. Trivella and R.E. Marques were responsible for laboratory analysis. R.R. Luiz, J.R. Lapa e Silva and P. Pelosi were responsible for the statistical analysis. K.G. Franchini, J.R. Lapa e Silva and P. Pelosi were also responsible for writing of the manuscript. All authors reviewed and approved the final version of the manuscript.

Conflict of interest: P.R.M. Rocco reports personal fees for data monitoring committee work from Sanofi, outside the submitted work. P.L. Silva has nothing to disclose. F.F. Cruz has nothing to disclose. M.A.C.M. Junior has nothing to disclose. P.F.G.M.M. Tierno has nothing to disclose. M.A. Moura has nothing to disclose. L.F.G. De Oliveira has nothing to disclose. C.C. Lima has nothing to disclose. E.A. Dos Santos has nothing to disclose. W.F. Junior has nothing to disclose. A.P.S.M. Fernandes has nothing to disclose. K.G. Franchini has nothing to disclose. E. Magri has nothing to disclose. N.F. de Moraes has nothing to disclose. J.M.J Gonçalves has nothing to disclose. M.N. Carbonieri has nothing to disclose. I.S. Dos Santos has nothing to disclose. N.F. Paes has nothing to disclose. P.V.M. Maciel has nothing to disclose. R.P. Rocha has nothing to disclose. A.F. de Carvalho has nothing to disclose. P.A. Alves has nothing to disclose. J.L.P. Modena has nothing to disclose. A.T. Cordeiro has nothing to disclose. D.B.B. Trivella has nothing to disclose. R.E. Marques has nothing to disclose. R.R. Luiz has nothing to disclose. P. Pelosi has nothing to disclose. J.R. Lapa e Silva has nothing to disclose.

Support statement: Supported by the Brazilian Council for Scientific and Technological Development (CNPq), Brazilian Ministry of Science, Technology, and Innovation for Virus Network; Brasília, Brazil, number: 403485/2020-7 and Funding Authority for Studies and Projects, Brasília, Brazil, number: 01.20.0003.00. Funding information for this article has been deposited with the Crossref Funder Registry.

\section{References}

1 Wu Z, McGoogan JM. Characteristics of and important lessons from the coronavirus disease 2019 (COVID-19) outbreak in China: summary of a report of 72314 cases from the Chinese Center for Disease Control and Prevention. JAMA 2020; 323: 1239-1242.

2 Mahmoud DB, Shitu Z, Mostafa A. Drug repurposing of nitazoxanide: can it be an effective therapy for COVID-19? J Genet Eng Biotechnol 2020; 18: 35.

3 Amadi B, Mwiya M, Musuku J, et al. Effect of nitazoxanide on morbidity and mortality in Zambian children with cryptosporidiosis: a randomised controlled trial. Lancet 2002; 360: 1375-1380.

4 Wang M, Cao R, Zhang L, et al. Remdesivir and chloroquine effectively inhibit the recently emerged novel coronavirus (2019-nCoV) in vitro. Cell Res 2020; 30: 269-271.

5 Speich B, Ame SM, Ali SM, et al. Efficacy and safety of nitazoxanide, albendazole, and nitazoxanide-albendazole against Trichuris trichiura infection: a randomized controlled trial. PLoS Negl Trop Dis 2012; 6: e1685. 
6 Broekhuysen J, Stockis A, Lins RL, et al. Nitazoxanide: pharmacokinetics and metabolism in man. Int J Clin Pharmacol Ther 2000; 38: 387-394.

7 Stockis A, Allemon AM, De Bruyn S, et al. Nitazoxanide pharmacokinetics and tolerability in man using single ascending oral doses. Int J Clin Pharmacol Ther 2002; 40: 213-220.

8 Stockis A, De Bruyn S, Gengler C, et al. Nitazoxanide pharmacokinetics and tolerability in man during 7 days dosing with $0.5 \mathrm{~g}$ and $1 \mathrm{~g}$ b.i.d. Int J Clin Pharmacol Ther 2002; 40: 221-227.

9 Walsh KA, Jordan K, Clyne B, et al. SARS-CoV-2 detection, viral load and infectivity over the course of an infection. J Infect 2020; 81: 357-371.

10 Wölfel R, Corman VM, Guggemos W, et al. Virological assessment of hospitalized patients with COVID-2019. Nature 2020; 581: 465-469.

11 Zheng S, Fan J, Yu F, et al. Viral load dynamics and disease severity in patients infected with SARS-CoV-2 in Zhejiang province, China, January-March 2020: retrospective cohort study. BMJ 2020; 369: m1443.

12 Pujadas E, Chaudhry F, McBride R, et al. SARS-CoV-2 viral load predicts COVID-19 mortality. Lancet Respir Med 2020; 8: e70.

13 de Wit E, van Doremalen N, Falzarano D, et al. SARS and MERS: recent insights into emerging coronaviruses. Nat Rev Microbiol 2016; 14: 523-534.

14 Cao B, Wang Y, Wen D, et al. A trial of lopinavir-ritonavir in adults hospitalized with severe COVID-19. N Engl J Med 2020; 382: 1787-1799.

15 Beigel JH, Tomashek KM, Dodd LE, et al. Remdesivir for the treatment of Covid-19 - final report. N Engl J Med 2020; 383: 1813-1826.

16 Goldman JD, Lye DCB, Hui DS, et al. Remdesivir for 5 or 10 days in patients with severe COVID-19. N Engl J Med 2020; 383: 1827-1837.

17 Hung IF, Lung KC, Tso EY, et al. Triple combination of interferon beta-1b, lopinavir-ritonavir, and ribavirin in the treatment of patients admitted to hospital with COVID-19: an open-label, randomised, phase 2 trial. Lancet 2020; 395: 1695-1704.

18 He X, Lau EHY, Wu P, et al. Temporal dynamics in viral shedding and transmissibility of COVID-19. Nat Med 2020; 26: 672-675.

19 Siddiqi HK, Mehra MR. COVID-19 illness in native and immunosuppressed states: a clinical-therapeutic staging proposal. J Heart Lung Transplant 2020; 39: 405-407.

20 Chang D, Mo G, Yuan X, et al. Time kinetics of viral clearance and resolution of symptoms in novel coronavirus infection. Am J Respir Crit Care Med 2020; 201: 1150-1152. 